\title{
Can Supraspinatus Tears Contribute to Acquired Subcoracoid Impingement? A Radiological Study of Anterosuperior Cuff Tears
}

\author{
M. J. Leite ${ }^{1}$, A. R. Pinho ${ }^{1,2,4}$, M. C. Sá ${ }^{3}$, M. R. Silva ${ }^{1}$, J. M. Almeida ${ }^{1}$, A. N. Sousa ${ }^{1}$, \\ J. M. Torres ${ }^{1,4}$ \\ 1 Orthopedics and Traumatology Service, São João University Hospital, Porto, Portugal \\ 2 Department of Biomedicine, Unit of Anatomy, Porto Medical School, Porto University, Center for Health \\ Technology and Services Research (CINTESIS), Porto, Portugal \\ 3 Primary Health Care Unit Saúde em Família, Porto, Portugal \\ 4 Porto Medical School, Porto University, Portugal
}

\section{CORRESPONDING AUTHOR:}

Maria J. Leite

Serviço de Ortopedia e Traumatologia

Centro Hospitalar Universitário de São João

Alameda Professor Hernani Monteiro 4200-319 Porto, Portugal

E-mail:mjlcma@gmail.com

DOI:

10.32098/mltj.02.2021.06

LEVEL OF EVIDENCE: $3 A$

\section{SUMMARY}

Backgroud. Many subscapularis (SubS) lesions represent the result of the progression of anterosuperior cuff tears, although their pathophysiology is still relatively unknown. The goal of this paper was to determine the influence of supraspinatus tears in the development of subcoracoid impingement and therefore SubS tears.

Methods. This is a retrospective, controlled and single-blinded study. We analyzed 301 patients with rotator cuff pathology and an MRI study. The coraco-humeral distance and coracoid overlap were measured. The presence of supraspinatus and subscapularis lesions were also evaluated.

Results. Supraspinatus tears were found to be associated with the presence of subscapularis tears $(p=0.003)$. A significant relationship $(p=0.002)$ was achieved between supraspinatus tears and inferior coraco-humeral distance, with average values of $8.4 \pm 2.9 \mathrm{~mm}$ and $10.2 \pm 3.2 \mathrm{~mm}$ in shoulders with and without supraspinatus tears respectively. On the opposite side, there wasn't a significant association between supraspinatus tears and the coracoid overlap.

Conclusions. This paper is, to our knowledge, the first to study the influence of supraspinatus tears in different parameters implied in subscapularis tears pathology, representing also the largest published sample on this theme, including 301 patients. Finally, this study offers an explanation for anterosuperior cuff progression pathophysiology, confirming that supraspinatus full thickness tears can lead to a secondary subcoracoid impingement and thus subscapularis tears.

\section{KEY WORDS}

Coracoid-humeral distance; coracoid overlap; rotator cuff tears; subscapularis; subcoracoid impingement; supraspinatus.

\section{BACKGROUND}

Rotator cuff tears pathophysiology remains controversial, with both probable contributions from degenerative processes, traumatic events and variants in shoulder anatomy (1-3). This is particularly true regarding subscapularis (SubS) tears, which pathophysiology and risk factors are still very much unknown $(1,2)$.
Although the understanding of degenerative rotator cuff pathology has evolved over the past decades, most of these studies focused on the more frequent posterosuperior cuff tears, including the infraspinatus and supraspinatus (SS) $(3,4)$. However, in recent years, there has been an increasing interest regarding anatomical risk factors for SubS tears, with the coracoacromial arch and the coracoid process being recognized as major players in anterior cuff tears $(2,5-7)$. Many 
papers have recently showed that subcoracoid impingement can lead to SubS tears, and that smaller coraco-humeral distances (CHD) and greater coracoid overlaps (CO) are associated with greater risk of anterior cuff pathology $(2,7)$. Despite the fact that many of SubS tears occur in the context of progression of a previous SS tear, there are relatively few studies focusing on the progression of anterosuperior tears, as described by Warner et al. (8), affecting the SS and SubS $(4,9)$.

Some studies have showed that SS tears are risk factors, and could progress to a SubS tear. This progression was particularly evident with full thickness SS tears, larger SS tears, and SS tears that included the anterior cable $(4,10)$.

Recently a few papers also re-established the previous notion that a rotator cuff tear would lead to the superior escape of the humeral head, because of the unopposed pull from the deltoid (10-12). Studies also agree that a superior cuff tear will lead to failing to compress the humeral head in the glenoid cavity, resulting in its superior migration $(10,12)$. On the other hand, papers have also showed that SubS tears do not cause a superior migration of the humeral head, more so, the addition of a SubS tear to a shoulder with previous SS tear didn't result in aggravated superior humeral head escape $(11,13)$.

However there is still a sparse number of papers focusing in the process behind the progression of anterosuperior tears, although studies agree that a superior cuff tear will result in superior humeral head migration which will result in a decreased coraco-humeral distance (CHD) $(10,12)$. This association between superior cuff complete tears and smaller $\mathrm{CHD}$, is explained by the superior migration of the humeral head, placing a part of the humeral head with greater cross section posteriorly to the coracoid, leading to a secondary subcoracoid impingement, defined by entrapment of the SubS between the coracoid process and the humerus (10, $12,14)$. In a study conducted by Nove-Josserand et al. (15), the authors reported an association between patients with SS, SubS and infraspinatus tears and diminished CHD (15). In a previous paper by MacMahon et al. (10), the authors compared the effect of complete SS tears in CHD and the association of these with SubS tears (10). They concluded that SS tears were associated with increased risk of SubS tears $(\mathrm{p}<0.05)$. They also found that patients with SS tendentially showed narrowing of the CHD, however without significant differences between the study and control group (10).

Similarly, to the subacromial impingement role in posterosuperior cuff tears, decreased CHD could be an etiologic factor in progression of SS tears anteriorly into the SubS $(7,12,16-18)$. Besides the CHD, studies also showed that the CO is also a very strong predictor of SubS tears (2). The CO is used to describe the coracoid shape as it measures the distance from the glenoid fossa to the most prominent aspect of the coracoid process (2). However, none of the previously published papers established if the presence of SS tears would influence the CO.

The main goal of this study was to evaluate the influence of SS full thickness tears in the CHD and CO. We postulated that a full thickness SS tear would result in a secondary subcoracoid impingement and help explain the pathophysiology of anterosuperior cuff tears.

\section{MATERIALS AND METHODS}

We performed a retrospective, controlled, single-blinded study. Patient data were collected retrospectively from our instituition's outpatient orthopedics clinical files, and included all patients with suspected rotator cuff pathology evaluated between 2009 and 2019. The study group included patients with full thickness SS tears, while the control group included patients with normal MRIs. Patients without an MRI study, with inflammatory arthropathy, rotator cuff tears other than full thickness SS tears, rotator cuff arthropathy, shoulder instability, or congenital deformities were excluded.

Our institution's standard MRI shoulder protocol was applied, including T1- and T2-weighted fat-saturated images, with the patient in supine position with the arm alongside the body, elbow extended, and forearm supinated. All MRI scans were performed in our institution's radiology department, using similar MRI models with equivalent gantries.

The CHD and CO were measured in transversal sections of T1 weighted images to take advantage of its better definition of the cortical margins. The CHD consists in the minimal distance between the humeral cortex and the coracoid cortex. The CO represents the distance from the glenoid to the tip of the coracoid process (figures 1, 2). The axial images were acquired where the subcoracoid space was at its minimum (5). The presence of SS tears, the presence and type of injury to the SubS and long head of the biceps (LHB), gender and laterality were also recorded.

A standardized measurement technique was developed, these measurements were recorded by the same orthopedic surgeon, blinded to the MRI report. The final recorded value was the average of three separated evaluations of each index.

\section{Statistical analysis}

Statistical analysis was performed using SPSS software (version 24; IBM, Armonk, NY, USA). Categorical variables are presented as absolute and relative frequencies, whereas continuous variables are characterized by the mean and 


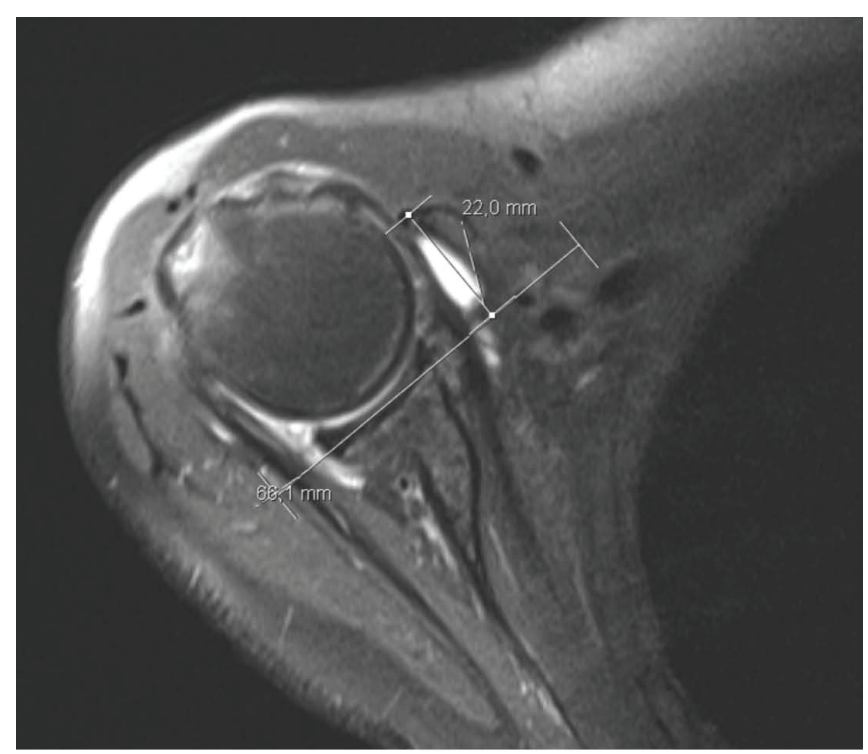

Figure 1. Coracohumeral distance measurement using T1-weighted MRI transversal sections.

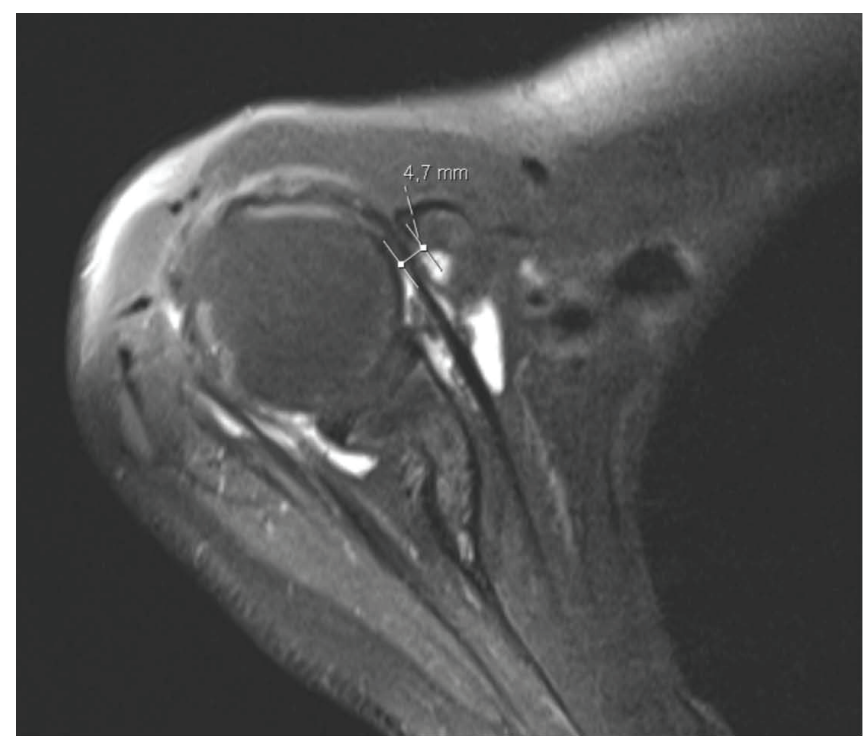

Figure 2. Coracoid overlap measurement using T1-weighted MRI transversal cuts.

standard deviation. The statistical tests used were the $\chi^{2}$ test to evaluate the association between categorical variables and 1-way analysis of variance test to compare means of continuous variables. The value with the highest Youden index was considered the cutoff with the best precision. Statistical significance was considered at $\mathrm{P}<.05$.

Ethical commission approval was obtained regarding this paper.

\section{RESULTS}

The sample was comprised of 301 shoulders, including 143 female $(47.5 \%)$ and 158 male $(52.5 \%)$. The study group included 272 shoulders with complete SS tears, and corresponded to $90.4 \%$ of the sample.

The sample included 145 shoulders with SubS injuries (tendinopathy or tear), corresponding to $48.2 \%$ of the sample, with $99(32.9 \%)$ presenting a SubS tear (complete or partial). Regarding the LHB, 93 shoulders presented a lesion of this tendon, including tendinopathy, rupture or instability.

The sample included 158 right $(52.5 \%)$ and 143 left shoulders $(47.5 \%)$. The left shoulder was associated with more SS tears $(\mathrm{p}=0.008)$.

Comparing the type of rotator cuff injuries in both genders, we didn't find any statistically significant relationship between the presence of a SS lesion and gender.

Analyzing the presence of simultaneous lesions of the SS, SubS or LHB, we found a significant association between SS lesions and lesions of the SubS $(\mathrm{p}=0.003)$ or LHB ( $\mathrm{p}$ $=0.045)$.

The CHD and CO were measured in 301 shoulders, with an average CHD of $8.6 \pm 3 \mathrm{~mm}$ and an average $\mathrm{CO}$ of 16.4 $\pm 4.8 \mathrm{~mm}$. There were no significant differences in CHD or $\mathrm{CO}$ variables according to gender. Regarding joint laterality, the CHD was significantly smaller in the left shoulder $(\mathrm{p}=$ 0.04); no statistical significance was obtained in the comparison between $\mathrm{CO}$ and joint laterality $(\mathrm{p}=0.34)$.

Comparing the $\mathrm{CHD}$ and $\mathrm{CO}$ with the presence of a SS lesion, we found that only the CHD showed a statistically significant association with the presence of a SS tear $(\mathrm{p}=$ 0.002). The mean CHD in the presence of a SS tear was 8.4 $\pm 2.9 \mathrm{~mm}$, contrasting with an average CHD value of $10.2 \pm$ $3.2 \mathrm{~mm}$ in the control group.

Regarding the CO, the average values were $16.5 \pm 4.7 \mathrm{~mm}$ and $15.1 \pm 4.7 \mathrm{~mm}$ in the shoulders with and without SS tears respectively. This difference didn't achieve statistical significance $(\mathrm{p}=0.12)$.

\section{DISCUSSION}

The role of subacromial and subcoracoid impingement in SS and SubS tears is extensively studied, however there are no published studies able to establish the exact pathophysiology of these two entities, that often are present simultaneously. In this paper we confirmed the relationship between the presence of SS full thickness tears and SubS tears, and even LHB injuries, with a statistical significant association between these lesions. Comparing both groups, we can see that the control group presents a healthy SubS in $82.8 \%$ of shoulders, and 
that only $48.5 \%$ of shoulders in the study group present a normal SubS. Besides that, $34.9 \%$ of the study group presented a SubS tear, contrasting with $13.7 \%$ in the control group. Regarding the CHD, we found a significant relationship between SS tears and decreased CHD, with average values of $10.2 \pm 3.2 \mathrm{~mm}$ and $8.4 \pm 2.9 \mathrm{~mm}$, for the control and study group respectively $(\mathrm{p}=0.002)$.

On the other hand, no statistical significant relationship was established between the CO and the presence of SS tears.

To our knowledge, our paper presents the largest sample on this topic, including 301 patients, being also the first to evaluate the different influence of the CHD as well as the CO in shoulders with SS tears and their roles in SubS tears pathology. However, there are some limitations to our study. Although our MRI protocol stated that the arm should be in a predefined position, variation in patient positioning is always a possibility and may influence the measurements. Also, our control group was relatively small when compared to the study group, due to the strict inclusion criteria for this group, which only included patients with completely normal MRI. In addition, our study was designed as a retrospective study, with its inherent limitations, and a prospective longitudinal study would be of value to confirm our preliminary conclusions.

It is known that many SubS tears originate from the anterior extension of SS tears, and many authors have already proved that SS tears are risk factors for SubS pathology $(3,9,10)$. More so, numerous papers have showed that SS tears result in superior migration of the humeral head with decreased acromio-humeral distance (10-12).

Differently, only a few study groups were able to establish the effect of SS tears in CHD, with complete SS tears associated with inferior $\mathrm{CHD}$, and none of these searched the effect on other known SubS predictors, such us CO $(10,12)$. Our results allow us to extrapolate that the humeral head superior migration results in a secondary subcoracoid impingement, resulting in increased risk of SubS tears in the context of SS full thickness tear.

As it is also known, both the $\mathrm{CO}$ and $\mathrm{CHD}$ are very strong predictors of SubS tears, with studies showing ROC Curves with areas under the curve of $93.8 \%$ and $80.6 \%$ (2). Both these parameters demonstrated being excellent SubS lesion

\section{REFERENCES}

1. Chalmers PN, Beck L, Granger E, Henninger H, Tashjian RZ. Superior glenoid inclination and rotator cuff tears. J Shoulder Elbow Surg 2018;27(8):1444-50.

2. Leite MJ, Sá M, Lopes MJ, Matos RM, Sousa AN, Torres JM. Coracohumeral distance and coracoid overlap as predictors of predictors in several studies, however they translate different anatomic particularities of the shoulder gridle. The CHD corresponds to the smaller distance between the humerus lesser tuberosity and the coracoid, and represents the space available for de SubS (2, 7, 19-21). This space can be altered by pathologies affecting the humerus lesser tuberosity or the coracoid, such as fracture mal union, osteophytes, cysts or anomalies of the lesser tuberosity, SS tendon calcifications or even abnormal coracoid anatomy $(2,7,19-21)$. As stated previously, other papers have also showed that the CHD can also be influenced by the presence of SS tears, and the phenom is explained by superior subluxation of the humeral head in these patients, that results in a greater humeral cross-section posteriorly to the coracoid process, and secondary subcoracoid impingement (10).

On the other hand, the CO represents the medial to lateral projection of the coracoid process, in regard to the glenoid fossa (2). This measurement is less susceptible to alterations caused by traumatic, iatrogenic or degenerative processes as it only depends on the coracoid projection, and the superior migration of the humeral head should not influence this measurement, as proved by our study.

The fact that the study group, with increased risk of SubS tears, was associated with inferior CHD but not with greater $\mathrm{CO}$, suggests that this subcoracoid impingement is secondary to subluxation of the humeral head and not a result of coracoid process morphology. This can represent one more clue in explaining the physiopathology of SubS tears as a progression of SS tears, in the context of anterosuperior rotator cuff tears.

Our results shed a light in anterior superior cuff progression pathophysiology, confirming the hypothesis that SS full thickness tears lead to a secondary subcoracoid impingement and consequently SubS tears. However future prospective longitudinal studies are needed to further explain this complex relationship. The study meets the ethical standards of the journal (22).

\section{CONFLICT OF INTERESTS}

The authors declare that they have no conflict of interests.

subscapularis and long head of the biceps injuries. J Shoulder Elbow Surg 2019;28:1723-7.

3. Olivia F, Piccirilli E, Bossa M, et al. I.S.Mu.L.T - Rotator Cuff Tears Guidelines. Muscles Ligaments Tendons J 2015;5(4):227-63. 
4. Mehta SK, Teefey SA, Middleton M, Steger-May K, Sefko JA, Keener JD. Prevalence and risk factors for development of subscapularis and biceps pathology in shoulders with degenerative rotator cuff disease: a prospective cohort evaluation. J Shoulder Elbow Surg 2020;29:451-8.

5. Gerber C, Terrier F, Ganz R. The role of the coracoid process in the chronic impingement syndrome. J Bone Joint Surg Br 1985;67(5):703-8.

6. Gerber C, Terrier F, Zehnder R, Ganz R. The subcoracoid space. An anatomic study. Clin Orthop Rel Res 1987;215:132-8.

7. Osti L, Soldati F, Buono A, Massari L. Subcoracoid impingement and subscapularis tendon: is there any truth? Muscles Ligaments Tendons J 2013;3(2):101-5.

8. Warner JJ, Higgins L, Parsons IM, Dowdy P. Diagnosis and treatment of anterosuperior rotator cuff tears. J Shoulder Elbow Surg 2001;10:37-46.

9. Lee J, Shukla DR, Sánchez-Sotelo J. Subscapularis tears: hidden and forgotten no more. JSES Open Access 2018;2(1):74-83.

10. MacMahon PJ, Taylor DH, Duke D, Brennan DD, O'Brien J, Eustace SJ. Contribution of full-thickness supraspinatus tendon tears to acquired subcoracoid impingement. Clin Radiol 2007;62(6):556-63.

11. Cetinkaya M, Ataoglu MB, Ozer M, Ayanoglu T, Oner AY, Kanatli U. Do subscapularis tears really result in superior humeral migration? Acta Orthop Traumatol Turc 2018;52(2):109-14.

12. Lo IK, Parten PM, Burkhart SS. Combined subcoracoid and subacromial impingement in association with anterosuperior rotator cuff tears: An arthroscopic approach. Arthroscopy 2003;19(10):1068-78.

13. Saupe N, Pfirrmann CW, Schmid MR, Jost B, Werner CM, Zanetti M. Association between rotator cuff abnormalities and reduced acromiohumeral distance. AJR Am J Roentgenol 2006;187:376e382.
14. Suenaga N, Minami A, Kaneda K. Postoperative subcoracoid impingement syndrome in patients with rotator cuff tear. J Shoulder Elbow Surg 2000;9:275-8.

15. Nove-Josserand L, Boulahia A, Levigne C, Noel E, Walch G. Coraco- humeral space and rotator cuff tears. Rev Chir Orthop Reparatrice Appar Mot 1999;85:677-83.

16. Bigliani LU, Cordasco FA, McIlveen SJ, Musso ES. Operative treatment of failed repairs of the rotator cuff. J Bone Joint Surg Am 1992;74:1505-15.

17. DeOrio JK, Cofield RH. Results of a second attemptat surgical repair of a failed initial rotator cuff repair. J Bone Joint Surg Am 1984;66:563-7.

18. Luo ZP, Hsu HC, Grabowski JJ, et al. Mechanical environment associated with rotator cuff tears. J Shoulder Elbow Surg 1998;7:616-20.

19. Abdrabou A, Shalaby M. Narrowed coraco-humeral distance on MRI: Association with subscapularis tendon tear. Egypt J Radiol Nucl Med 2017;48(4):977-81.

20. Çetinkaya M, Baybars M, Ataoglu B, Mustafa O, Ayanoglu T, Kanatli U. Subscapularis Tendon Slip Number and Coracoid Overlap are More Related Parameters for Subcoracoid Impingement in Subscapularis Tears: A Magnetic Resonance Imaging Comparison Study. Arthroscopy 2016;1-9.

21. Hekimoğlu B, Aydın H, Kızılgöz V, Tatar IG, Ersan O. Quantitative measurement of humero-acromial, humero-coracoid, and coraco-clavicular intervals for the diagnosis of subacromial and subcoracoid impingement of shoulder joint. Clin Imaging. 2013;37(2):201-10.

22. Padulo J, Oliva F, Frizziero A, Maffulli N. Muscles, Ligaments and Tendons Journal - Basic principles and recommendations in clinical and field Science Research: 2018 update. Muscles Ligaments Tendons J 2018;8(3):305-7. 